\title{
A SPECIAL CASE OF SCHÖNFLIES THEOREM FOR QUASICONFORMAL MAPPINGS IN $n$-SPACE
}

\author{
DAVID B. GAULD and M. K. VAMANAMURTHY
}

1. Introduction. Gehring [5] first proved extension theorems for quasiconformal mappings in $n$-space using the ideas of Mazur [7] and Morse [8]. We have recently [4] given an alternative proof of Gehring's Theorem 1 [5] using the ideas of Brown [2]. Näätänen [9] has obtained estimates for the dilatation of Gehring's extension under an additional hypothesis. In this note we construct an extension for this special case, obtain estimates for the dilatation of the extended mapping and finally apply this to the case of an important plane mapping as in [9].

We thank Professor Gehring for helpful discussions. The work was completed during the second author's visit to the University of Michigan while on leave from the University of Auckland. Thanks are due to both the Universities.

2. Notation. We let $\boldsymbol{R}^{n}, \overline{\boldsymbol{R}}^{n}=\boldsymbol{R}^{n} \cup\{\infty\}$, denote, respectively, the $n$-dimensional $(n \geqq 2)$ euclidean and Möbius spaces. For $x=\left(x_{1}, x_{2}, \ldots, x_{n}\right)=\sum_{j=1}^{n} x_{j} e_{j} \in \boldsymbol{R}^{n}$, we let $|x|$ denote the usual euclidean norm. In $\boldsymbol{R}^{2}$ we also use the complex notation $z=x_{1}+i x_{2}, i=\sqrt{-1}$. For $E \subset \overline{\boldsymbol{R}}^{n}$ we let $\bar{E}, \partial E$, int $E$, denote, respectively, the closure, boundary, and interior of $E$, with respect to $\overline{\boldsymbol{R}}^{n}$. For $a \in \boldsymbol{R}^{n}, r>0$, $B^{n}(a ; r)$ is the open ball with center $a$, radius $r$ and $S^{n-1}(a ; r)=\partial B^{n}(a ; r)$. In particular, $B^{n}(r)=B^{n}(0 ; r), B^{n}=B^{n}(1), S^{n-1}(r)=S^{n-1}(0 ; r), S^{n-1}=S^{n-1}(1)$. For $p=0,1,2, \ldots$, we let $\omega_{p}$ denote the $p$-dimensional Lebesgue measure of $S^{p}$. We observe that $\omega_{0}=2, \omega_{1}=2 \pi, \omega_{2}=4 \pi, \ldots$ and in general, $\omega_{p}=$ $=2 \pi^{(p+1) / 2} / \Gamma((p+1) / 2)$, where $\Gamma$ denotes the classical Gamma function. For other definitions and notations we refer to [11].

\section{Results.}

Theorem 1. Let $\bar{B}_{2} \subset B_{1}, \bar{D}_{2} \subset D_{1}$, where $B_{1}, B_{2}, D_{2}$ are balls and $D_{1}, a$ Jordan domain in $\overline{\boldsymbol{R}}^{n}$. Let $B=B_{1}-\bar{B}_{2}, A=D_{1}-\bar{D}_{2}$ and $f: \bar{B} \rightarrow \bar{A}$, a homeomorphism such that $f\left(\partial B_{i}\right)=\partial D_{i}, i=1,2$. Then there exists a homeomorphism $F$ of $\bar{B}_{1}$ onto $\bar{D}_{1}$ such that $F(x)=f(x)$, for all $x$ in $\bar{B}$. Further, if $f$ is quasiconformal, then so is $F$. 

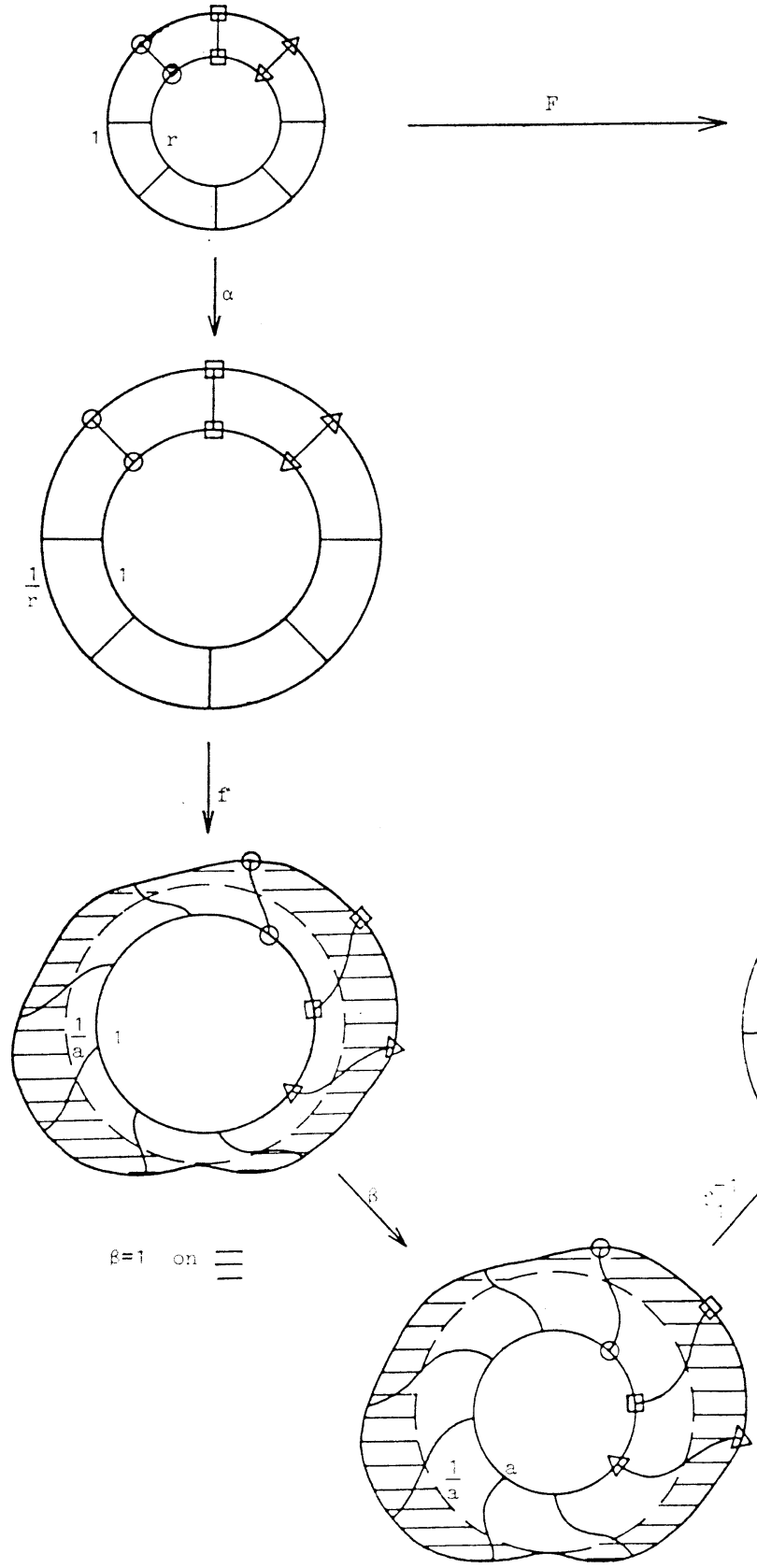
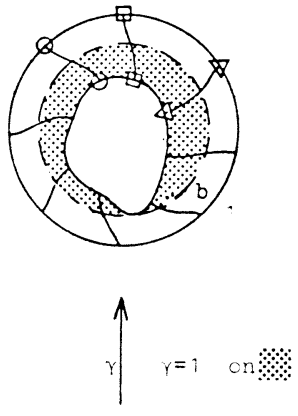
Proof. By performing preliminary Möbius transformations we may assume that $B_{1}=B^{n}(1 / r), 0<r<1$, and $B_{2}=D_{2}=B^{n}$. We let $F(x)=f(x)$, for $x$ in $\bar{B}_{1}-B_{2}$. Next, to define $F$ on $B_{2}$, we use a construction (see the figure) similar to that of Lemma 9 in [4]. Let $f_{1}$ be the reflection of $f$ in $\partial B_{2}$, that is

Let

$$
f_{1}(x)=\left\{\begin{array}{l}
f(x) \text { for } 1 \leqq|x| \leqq 1 / r \\
f\left(x|x|^{-2}\right)\left|f\left(x|x|^{-2}\right)\right|^{-2} \text { for } r \leqq|x| \leqq 1
\end{array}\right.
$$

$$
\begin{array}{ll}
a & =\max \left\{\left|f_{1}(x)\right|: \quad|x|=r\right\} \\
b & =\max \left\{\left|f_{1}^{-1}(x)\right|:|x|=a\right\} .
\end{array}
$$

Let $\alpha, \beta$ and $\gamma$ be radial mappings of $\boldsymbol{R}^{n}$ defined as follows:

$$
\begin{gathered}
\alpha(x)=x / r, \\
\beta(x)=\left\{\begin{array}{lll}
a x|x| & \text { for }|x| \leqq 1 / a, \\
x & \text { for } & |x| \geqq 1 / a,
\end{array}\right. \\
\gamma(x)=\left\{\begin{array}{lll}
r^{\frac{\log b}{\log (r b)}}|x|^{-\frac{\log r}{\log (r b)}} x & \text { for }|x| \geqq b, \\
x & \text { for }|x| \leqq b .
\end{array}\right.
\end{gathered}
$$

Define the mapping $F$ on $\bar{B}_{2}-B^{n}(r)$ by

$$
F=f_{1} \circ \gamma \circ f_{1}^{-1} \circ \beta \circ f \circ \alpha .
$$

Then $F(x)=f(x)$ for $x \in \partial B_{2}$ and $F\left(S^{n-1}(r)\right)=S^{n-1}(a)$.

Finally, the mapping $F$ on $\bar{B}^{n}(r)$ is obtained by reflection. Moreover, if $f$ is quasiconformal, so is $F$, since all the auxiliary mappings are quasiconformal.

In the next theorem we obtain estimates for the maximal dilatation of the above extension $F$.

Theorem 2. Let the mappings $f$ and $F$ be as in Theorem 1 and let $K(f)=K$. Then $K(F)=K^{*}$ satisfies

$$
K \leqq K^{*} \leqq K^{3}(2 M)^{n-1} C^{2^{1-n} C^{M^{1-n}},}
$$

where

$$
\begin{gathered}
C=C(n, K)=\exp \left((n-1) K \frac{\omega_{n-1}}{\omega_{n-2}} q^{n-1}\right), \\
M=\bmod B=\log \frac{1}{r}, \quad \text { and } \quad q=\int_{0}^{\pi / 2}(\sin t)^{\frac{2-n}{n-1}} d t .
\end{gathered}
$$

Moreover, for large values of $M$, (i.e. $r \rightarrow 0$ ), we have

$$
K^{*} \leqq 4^{n-1} K^{5}\left(\frac{M}{M-\left(K^{2 /(n-1)}+K^{1 /(n-1)}\right) \log \lambda}\right)^{n-1},
$$

where $\lambda=\lambda(n)$ is a positive constant. 
Proof. Let the notation be as in Theorem 1 . Since $F$ extends $f$, clearly $K \leqq K^{*}$. Next, $\quad K\left(f_{1}\right)=K(f)=K, \quad K(\alpha)=1, \quad K(\beta)=2^{n-1}, \quad$ and $\quad K(\gamma)=(\log (r b) / \log b)^{n-1}$. Thus

$$
K \leqq K^{*} \leqq 2^{n-1} K^{3}(\log (r b) / \log b)^{n-1} \leqq 2^{2(n-1)} K^{3} M^{n-1}(\log (1 / b))^{1-n} .
$$

The result follows once we eliminate $b$ in the above inequality. For this we show that

$$
\log \frac{1}{b} \geqq 2 \exp \left(-K C_{1} \cdot 2^{1-n} \exp \left((n-1) K C_{1} M^{1-n}\right)\right),
$$

and

$$
\log \frac{1}{b} \geqq M K^{\frac{2}{1-n}}-\left(1+K^{\frac{1}{1-n}}\right) \log \lambda,
$$

where $C_{1}=C_{1}(n)$ and $\lambda=\lambda(n)$ are positive constants. We observe that (5) is nontrivial only when $M$ is so large that the right side of (5) is positive.

By the extremal property of the Grötzsch ring [3] we get

$$
\log \frac{1}{a} \leqq K^{\frac{1}{n-1}} \bmod R_{G}(b),
$$

where $R_{G}(b)$ is the ring

$$
B^{n}-\left\{t e_{1}: 0 \leqq t \leqq b\right\} .
$$

Next by Lemma 9.9 in [3] and Corollary 1 in [1] we get

$$
\log \frac{1}{a} \leqq K^{\frac{1}{n-1}} \min \left[\log \frac{\lambda}{b},\left(\frac{C_{1}}{\log ((1+b) /(1-b))}\right)^{\frac{1}{n-1}}\right],
$$

where $\lambda=\lim _{t \rightarrow 0} t \exp \left(\bmod R_{G}(t)\right)$ and $C_{1}=\left(\omega_{n-1} / \omega_{n-2}\right) q^{n-1}$.

Similarly

From (7),

$$
M=\log \frac{1}{r} \leqq K^{\frac{1}{n-1}} \min \left[\log \frac{\lambda}{a},\left(\frac{C_{1}}{\log ((1+a) /(1-a))}\right)^{\frac{1}{n-1}}\right] .
$$

hence

$$
\log \frac{1+b}{1-b} \leqq \frac{K C_{1}}{(\log (1 / a))^{n-1}},
$$

$$
\log \frac{1}{b} \geqq \log \operatorname{coth} \frac{K C_{1}}{2(\log (1 / a))^{n-1}} \geqq 2 \exp \left(-\frac{K C_{1}}{(\log (1 / a))^{n-1}}\right)
$$

since $\log \operatorname{coth} x \geqq 2 \exp (-2 x)$ for $x>0$. Similarly from (8) we have

so that

$$
\log \frac{1}{a} \geqq 2 \exp \left(-\frac{K C_{1}}{M^{n-1}}\right) \text {, }
$$

$$
\log \frac{1}{b} \geqq 2 \exp \left(-K C_{1} \cdot 2^{1-n} \exp \left(K C_{1} M^{1-n}(n-1)\right)\right),
$$

which is (4). 
Next (5) follows from (7) and (8). Now (3) and (4) imply (1) while (3) and (5) yield (2). This completes the proof of Theorem 2 .

Remark. The construction of $F$ in the proof of Theorem 1 does not require $f$ to be one to one on $S^{n-1}(1 / r)$ provided it extends to a homeomorphism of $f^{-1}\left(\bar{B}^{n}(1 / a)\right)-B_{2}$ onto $\bar{B}^{n}(1 / a)-D_{2}$. Hence under these conditions the construction of $F$ is valid even when $f\left(\partial B_{1}\right)$ is not the boundary of a Jordan domain. In particular, on reflection, the construction applies to the case where $n=2$ and $f$ is the conformal mapping of the plane annulus $r<|z| \leqq 1$ onto the ring $\bar{B}_{2}$ minus the slit $[-a, a]$ of the real axis [10].

Theorem 3. Let $f$ be the above plane conformal mapping and $F$ the extension given by the proof of Theorem 1. Then the maximal dilatation $K^{*}$ of $F$ satisfies

and

$$
K^{*} \leqq 2 M C^{(1 / 2) C^{1 / M}} \text { for } 0<r<1,
$$

$$
K^{*} \leqq \frac{4 M}{M-\log 16} \quad \text { for } \quad 0<r<\frac{1}{16}
$$

where $M=\log (1 / r), C=\exp \left(\pi^{2} / 2\right)$.

Proof. Follows from (1) and (2) by putting $n=2, K=1$ and $\lambda=4$ (see [6]).

Remark. Given a quasiconformal mapping $f$ as in Theorem 1 , let $\hat{R}(f)$ denote the infimum of the dilatations of all possible quasiconformal extensions $F$ of $f$ satisfying Theorem 1. In [9], Näätänen defines the function $\varphi(n, K, M)$ to be the supremum of the numbers $\hat{K}(f)$ as $f$ ranges through all mappings with dilatation $\leqq K$ and shows that it is a decreasing function of $M$. The estimates in Theorem 2 show that $K \leqq \lim _{M \rightarrow \infty} \varphi(n, K, M) \leqq 4^{n-1} K^{5}$. However, as $M \rightarrow 0$, the upper bound for $\varphi(n, K, M)$ in [9] is better than that in our Theorem 2.

\section{References}

[1] Anderson, G. D.: Extremal rings in $n$-space for fixed and varying $n$. - Ann. Acad. Sci. Fenn. Ser. A I $575,1974,1-21$.

[2] Brown, M.: A proof of the generalized Schönflies theorem. - Bull. Amer. Math. Soc. 66, 1960, 74-76.

[3] Caraman, P.: $n$-dimensional quasiconformal mappings. - Abacus Press, Tunbridge Wells, Kent, England, 1974.

[4] Gauld, David B., and M. K. Vamanamurthy: Quasiconformal extensions of mappings in n-space. - Ann. Acad. Sci. Fenn. Ser. A I 3, 1977, 229-246.

[5] Gehring, F. W.: Extension theorems for quasiconformal mappings in $n$-space. - J. Analyse Math. 19, 1967, 149-169.

[6] Lehto, O., and K. I. VIRTANEN: Quasiconformal mappings in the plane. - Springer-Verlag, Berlin-Heidelberg-New York, 1973.

[7] Mazur B.: On embeddings of spheres. - Acta Math. 105, 1961, 1-17. 
[8] Morse, M.: A reduction of the Schönflies extension problem. - Bull. Amer. Math. Soc. 66, 1960, 113-115.

[9] NÄÄTÄNEN, M.: Dilatation estimates for quasiconformal extensions. - Israel J. Math. (to appear).

[10] NehaRI, Z.: Conformal mapping. - McGraw-Hill Book Co., Inc., New York-Toronto-London, 1952.

[11] VÄISÄLÄ, J.: Lectures on $n$-dimensional quasiconformal mappings. - Lecture Notes in Mathematics 229, Springer-Verlag, Berlin-Heidelberg-New York, 1971.

University of Auckland

Department of Mathematics

Auckland

New Zealand
University of Michigan

Department of Mathematics

Ann Arbor, Michigan 48109 USA

University of Auckland

Department of Mathematics

Auckland

New Zealand

Received 7 November 1977 